\title{
Accuracy of childhood asthma control test among Thai childhood asthma patients
}

\author{
Sirasuda Sommanus, ${ }^{1}$ Chalerat Direkwattanachai, ${ }^{2}$ Saranath Lawpoolsri, ${ }^{3}$ Raweerat Sitcharungsi ${ }^{4}$
}

\begin{abstract}
Background: The Childhood Asthma Control Test (C-ACT) was developed to assess asthma control in children worldwide. A self-administered questionnaire for children translated into Thai language was used.

Objective: To validate the C-ACT cut-points for evaluating the level of asthma control among Thai children, using the Global Initiative for Asthma (GINA) guideline as a gold standard.

Methods: C-ACT score, FEV1 and assessment of level of asthma control were recorded at baseline, 3-month, 6-month, and 1-year visits among children with asthma. Receiver operating characteristic (ROC) curves was used to determine the area under the curve (AUC) of C-ACT score for determining the level of asthma control. Validity indicators were calculated at different C-ACT cut-points to determine those most appropriate for predicting controlled and uncontrolled asthma.

Results: We enrolled 279 children, $64 \%$ males, with mean age $6.87 \pm 2.4$ years. C-ACT score was significantly correlated with FEV1 at 3 -month, 6 -month, and 1 -year visits $(\mathrm{p}<0.001)$. The AUC of C-ACT score compared with GINA score were above $80 \%$ at all visits. The suggested C-ACT score cut-point of controlled asthma was $\geq 23$ (sensitivity $69.5 \%$, specificity $73.3 \%$, positive predictive value (PPV) $81.2 \%$, negative predictive value (NPV) $63.8 \%$ ); that of uncontrolled asthma was $\leq$ 18 (sensitivity $54.2 \%$, specificity $96.9 \%$, PPV 61.9\%, NPV 95.7\%).
\end{abstract}

Conclusions: The Thai version of the C-ACT is an accurate, simple, and useful tool for assessing asthma control among Thai children. The high AUC suggests that the Thai C-ACT is as good as the GINA guideline in predicting asthma control level.

Keywords: Accuracy, Asthma, Childhood asthma control test, Global Initiative for Asthma guidelines, FEV1

\section{From:}

${ }^{1}$ Department of Pediatrics, Taksin Hospital, Bangkok, Thailand

${ }^{2}$ Department of Pediatrics, Faculty of Medicine Ramathibodi Hospital, Mahidol University, Bangkok, Thailand

${ }^{3}$ Department of Tropical Hygiene, Faculty of Tropical Medicine, Mahidol University, Bangkok, Thailand

${ }^{4}$ Department of Tropical Pediatrics, Faculty of Tropical Medicine, Mahidol University, Bangkok, Thailand

Corresponding author:

Raweerat Sitcharungsi

Faculty of Tropical Medicine, Mahidol University, Bangkok 10400, Thailand

Email: raweeratsitcharungsi@gmail.com

\section{Abbreviations:}

$\mathrm{C}-\mathrm{ACT}=$ Childhood asthma control test

FEV1 $=$ Forced expiratory volume in one second

GINA = Global Initiative for Asthma

DSS $=$ Disease severity score

AUC $=$ Area under the curve

ROC $=$ Receiver operating characteristic

$\mathrm{PPV}=$ Positive predictive value

$\mathrm{NPV}=$ Negative predictive value

\section{Background}

Childhood asthma is caused by chronic inflammation, which leads to airway hyper-reactivity, recurrent wheezing, and persistently altered airway function. Asthma is a common disease, affecting approximately $1 \%-18 \%$ of people worldwide. ${ }^{1}$ The International Study of Asthma and Allergies in Childhood (ISAAC) Phase Three reported that the prevalence of current asthma in children aged 13-14 years was 13.8\%, and it was $11.6 \%$ in those aged 6-7 years. According to that study, the reported lifetime prevalence of asthma in Thailand was $12.3 \%{ }^{2}$

Well-controlled asthma requires early diagnosis and proper assessment of asthma control to provide appropriate treatment. A diagnosis of asthma should be given based on patient history, physical examination, and pulmonary function tests, such as forced expiratory volume in 1 second (FEV1), peak flow variability or a bronchial provocation test. ${ }^{3}$ The goal of asthma therapy is to control symptoms and minimize future risk of exacerbation, decline of lung function and 
adverse outcomes. Assessment and monitoring of the level of asthma control are important. According to the Global Initiative for Asthma (GINA) 2017 guideline, the level of asthma control is classified into well-controlled, partly controlled, and uncontrolled asthma groups. The level of asthma control can be determined by daytime and nighttime symptoms, use of rescue medication, limitation of activities, exacerbation, and pulmonary function tests. ${ }^{1}$ Pulmonary function testing is usually not available in the primary care setting. Worldwide asthma organizations have developed simplified questionnaires to assess asthma control with no equipment required, to be used in limited resource settings. Validated questionnaires, such as the Childhood Asthma Control Test (C-ACT) ${ }^{4}$ and Disease Severity Score (DSS), ${ }^{5}$ have been adopted and are widely used in primary care units worldwide.

The C-ACT was developed to assess childhood asthma control using a self-administered questionnaire with seven items. The questionnaire is divided into two parts. The first four items contain questions to be answered by children aged 4-11 years. The last three items are questions to be answered by parents or caregivers. This questionnaire assesses daytime and nighttime asthma symptoms, use of reliever medication, and limitations of daily activities in the prior 4 weeks. A previous C-ACT study found that scores of more than 19 indicated controlled asthma. ${ }^{4}$ The C-ACT can be used as an indicator to evaluate change in the clinical status of children with asthma. The C-ACT has been translated into different languages to be used worldwide. The cut-points of the C-ACT score for determining the level of asthma control can vary across countries, owing to variations in patient characteristics. ${ }^{7-15}$ The Thai version of the C-ACT has been used for decades in Thailand. However, appropriate cut-points of the C-ACT for Thai childhood asthma patients remain uncertain. Therefore, this study was conducted to determine the appropriate C-ACT score cut-points for evaluation of controlled, partly controlled, and uncontrolled asthma among Thai children.

\section{Methods \\ Study population}

Retrospective cohort study was conducted at a pediatric allergy unit at Taksin Hospital in Bangkok, Thailand during April 2012 to April 2016 were reviewed. The inclusion criteria were newly diagnosed asthma patients aged 4-14 years at enrollment. An asthma diagnosis was based on clinical symptoms and the GINA guideline. We excluded those patients who were unable to carry out the pulmonary function test (spirometry) or had underlying diseases including other chronic lung diseases, central nervous system diseases, cardiovascular diseases, and other chronic illnesses.

At diagnosis, each patient was evaluated by a pulmonary function test and asthma severity assessment according to the GINA guideline. C-ACT scores and FEV1 values were recorded at 3-month, 6-month, and 1-year visits, and assessment of the level of asthma control according to the GINA guideline was carried out at each visit. A Thai version of the C-ACT was developed by translating the original one by a linguist. To better understand patients' feelings about their asthma and facilitate good communication between clinicians and patients, the validated version of C-ACT in Thai language was used. Spirometry was performed according to American Thoracic Society standards. ${ }^{6}$

\section{Ethical approval}

This study was approved by the Bangkok Metropolitan Administration Ethics Committee for Human Research (BMAEC-S010q/59_EXP). Since this study involved only retrospectively review medical records, informed consent of each participants was not required.

\section{Statistical analysis}

Demographic and clinical characteristics of enrolled patients at baseline were described. C-ACT scores and FEV1 values were summarized as mean and standard deviation (SD) at each visit. An ANOVA test was performed to determine the difference in the mean among the three levels of asthma control. Then, the correlation between C-ACT score and FEV1 was determined at each visit, using a Pearson correlation test. The level of asthma control at each visit was assessed according to the GINA guideline (gold standard). Three levels of asthma control were designated: controlled, partly controlled, and uncontrolled. The validity of the C-ACT score to predict controlled and uncontrolled asthma was measured according to the GINA guideline. The receiver operating characteristic (ROC) was used to determine the ability of using C-ACT score to predict asthma control, indicated by the area under the curve (AUC). In addition, validity indicators of the test including sensitivity, specificity, positive predictive value $(\mathrm{PPV})$, and negative predictive value (NPV) were calculated to determine the appropriate C-ACT cut-points. The number of patients with uncontrolled asthma decreased overtime after receiving treatment: therefore, the validity indicators were evaluated only at the 3 -month visit.

To determine appropriate cut-points of C-ACT scores, the clinical validity of potential C-ACT cut-points was evaluated by comparison with the percentage of predicted FEV1 value $(<60 \%, 60 \%$ to $79 \%, 80 \%$ to $99 \%$, and $\geq 100 \%)$, the level of control according to the GINA guideline, and the change in medical therapy (step up, no change, and step down) that the patient received.

\section{Results \\ Subject characteristics}

Overall, 279 children were enrolled. Among these, 179 patients $(64 \%)$ were male and the mean age was $6.87 \pm 2.4$ years. The mean age at asthma diagnosis was $4.88 \pm 2.31$ years and age at onset was $3.31 \pm 2.05$ years. According to severity, most children had moderate persistent (131; 46.6\%), mild persistent $(70 ; 25.1 \%)$, mild intermittent $(30 ; 10.7 \%)$, and severe persistent $(48 ; 17.6 \%)$ asthma. During the 1 year of follow-up, 15 children were lost to follow-up at the 6-month visit; a total 230 children $(82.4 \%)$ completed a 1 -year visit. The most common comorbidities were allergic rhinitis (40.5\%), snoring $(20.1 \%)$, and atopic dermatitis (14.7\%). More than half of patients had a history of potential risk factors for asthma, including history of hospitalization owing to lower respiratory 
tract infection $(58.4 \%)$, family history of atopy (52.3\%), and passive smoking (50.5\%), as shown in Table 1. According to the GINA guideline, 174 (62.4\%), 81 (29\%), and 24 (8.6\%) patients were categorized as having controlled, partly controlled, and uncontrolled asthma, respectively.

Table 1. Patient baseline clinical characteristics at the first visit.

\begin{tabular}{|lll}
\hline Characteristics & Number & Percentage \\
\hline Total number & $\mathrm{N}=279$ & $100 \%$ \\
\hline Male & 179 & $64.2 \%$ \\
\hline Female & 100 & $35.8 \%$ \\
\hline Characteristics N=279 & Years & Range \\
\hline Age (year), mean \pm SD & $6.87 \pm 2.49$ & $(4-14.2)$ \\
\hline Age of diagnosis (year), mean \pm SD & $4.88 \pm 2.31$ & $(1-12)$ \\
\hline Age of onset (year), mean \pm SD & $3.31 \pm 2.05$ & $(0.5-10)$ \\
\hline $\begin{array}{l}\text { Duration of disease before diagnosis (year), } \\
\text { mean } \pm \text { SD }\end{array}$ & $1.57 \pm 1.35$ & $(0-8)$ \\
\hline Severity of asthma N=279 & Number & Percentage \\
\hline Mild intermittent $(>80 \%$ predicted FEV1) & 30 & $10.7 \%$ \\
\hline Mild persistent $(>80 \%$ predicted FEV1) & 70 & $25.1 \%$ \\
\hline $\begin{array}{l}\text { Moderate persistent }(60-80 \% \text { predicted } \\
\text { FEV1) }\end{array}$ & 131 & $46.6 \%$ \\
\hline Severe persistent $(<60 \%$ predicted FEV1) & 48 & $17.6 \%$ \\
\hline Co-morbid diseases N=279 & Number & Percentage \\
\hline Allergic rhinitis & 113 & $40.5 \%$ \\
\hline Atopic dermatitis & 41 & $14.7 \%$ \\
\hline Food allergy & 19 & $6.8 \%$ \\
\hline $\begin{array}{l}\text { History of sinusitis } \\
\text { tract infection }\end{array}$ & 37 & $13.3 \%$ \\
\hline History of urticarial rash & 14 & $5 \%$ \\
\hline Snoring & 56 & $20.1 \%$ \\
\hline
\end{tabular}

Abbreviations: SD, standard deviation; FEV1, Forced expiratory volume in 1 second.

\section{Correlation between C-ACT score and FEV1}

Table 2 shows the mean (SD) C-ACT score and FEV1 at 3-month, 6-month, and 1-year visits for the controlled, partly controlled, and uncontrolled asthma groups. The means of C-ACT score and FEV1 were significantly different among the three asthma control levels. A significantly positive correlation between $\mathrm{C}$-ACT score and FEV1 was found at the 3-month ( $\mathrm{r}$ $=0.48$ and $\mathrm{p}<0.001), 6$-month $(\mathrm{r}=0.558$ and $\mathrm{p}<0.001)$, and 1 -year visits $(\mathrm{r}=0.421$ and $\mathrm{p}<0.001)$.

\section{Validity of C-ACT score}

According to the ROC, C-ACT scores showed good performance in predicting controlled and uncontrolled asthma, using GINA guideline classification as the gold standard. The AUC of C-ACT score was above $80 \%$ and $90 \%$ for detection of controlled and uncontrolled asthma, respectively, at all follow-up visits. The AUC of C-ACT score for controlled asthma was $0.81(0.76-0.87), 0.84(0.78-0.90)$, and $0.82(0.75-$ $0.90)$ for 3-month, 6-month, and 1-year visits, respectively. The AUC of C-ACT score for uncontrolled asthma was 0.93 (0.89-0.97), 0.91 (0.85-0.97), and 0.92 (0.87-0.97) for 3-month, 6-month, and 1-year visits, respectively.

A C-ACT score of 20-24 indicated controlled asthma at the 3-month visit, with sensitivity ranging from $47.7 \%$ to $95.4 \%$ and specificity ranging from $44.8 \%$ to $87.6 \%$ (Figure 1),(Table 3). A cut-point value of $\geq 23$ demonstrated $69.5 \%$ sensitivity, $73.3 \%$ specificity, $81.2 \% \mathrm{PPV}$, and $63.8 \% \mathrm{NPV}$. A C-ACT score of 17-21 indicated uncontrolled asthma, with sensitivity ranging from $45.8 \%$ to $91.7 \%$ and specificity ranging from $78.8 \%$ to $99.2 \%$ (Figure 1). A cut-point value of

Table 3. Sensitivity, specificity, positive predictive value, and negative predictive value of C-ACT scores to detect controlled asthma.

\begin{tabular}{ccccc}
\hline C-ACT $(\geq)$ & Sensitivity & Specificity & PPV (\%) & NPV (\%) \\
\hline 20 & 0.954 & 0.448 & 74.11 & 85.45 \\
21 & 0.902 & 0.562 & 77.34 & 77.63 \\
22 & 0.828 & 0.638 & 79.12 & 69.07 \\
\hline 23 & $\mathbf{0 . 6 9 5}$ & $\mathbf{0 . 7 3 3}$ & $\mathbf{8 1 . 2 1}$ & $\mathbf{6 3 . 8 1}$ \\
24 & 0.477 & 0.876 & 82.76 & 59.42 \\
\hline
\end{tabular}

Abbreviations: C-ACT, Childhood Asthma Control Test; PPV, positive predictive value; NPV, negative predictive value.

Table 2. Mean(SD) of C-ACT scores and FEV1 values at all visits.

\begin{tabular}{|c|c|c|c|c|c|c|c|c|c|}
\hline & \multicolumn{3}{|c|}{ At 3 months } & \multicolumn{3}{|c|}{ At 6 months } & \multicolumn{3}{|c|}{ At 1 years } \\
\hline & $\mathrm{N}(279)$ & FEV1 & C-ACT & $\mathrm{N}(264)$ & FEV1 & C-ACT & $\mathrm{N}(230)$ & FEV1 & C-ACT \\
\hline Controlled & 174 & $94.39(12.97)$ & $24.15(1.84)$ & 185 & $93.61(12.20)$ & $24.32(1.60)$ & 176 & $93.77(12.82)$ & $24.68(1.71)$ \\
\hline Partly controlled & 81 & $72.66(7.94)$ & $21.89(2.47)$ & 62 & $71.66(8.22)$ & $21.29(17.53)$ & 46 & $73.50(6.29)$ & $21.89(2.69)$ \\
\hline Uncontrolled & 24 & $57.23(8.49)$ & $18.08(2.50)$ & 17 & $58.20(7.78)$ & $17.53(3.84)$ & 8 & $55.48(8.32)$ & $19.25(2.61)$ \\
\hline p-value* & & $<0.001$ & $<0.001$ & & $<0.001$ & $<0.001$ & & $<0.001$ & $<0.001$ \\
\hline
\end{tabular}

${ }^{\star}$ ANOVA p-value comparing the different levels of asthma control.

Abbreviations: C-ACT, Childhood Asthma Control Test; SD, standard deviation; FEV1, Forced expiratory volume in 1 second. 

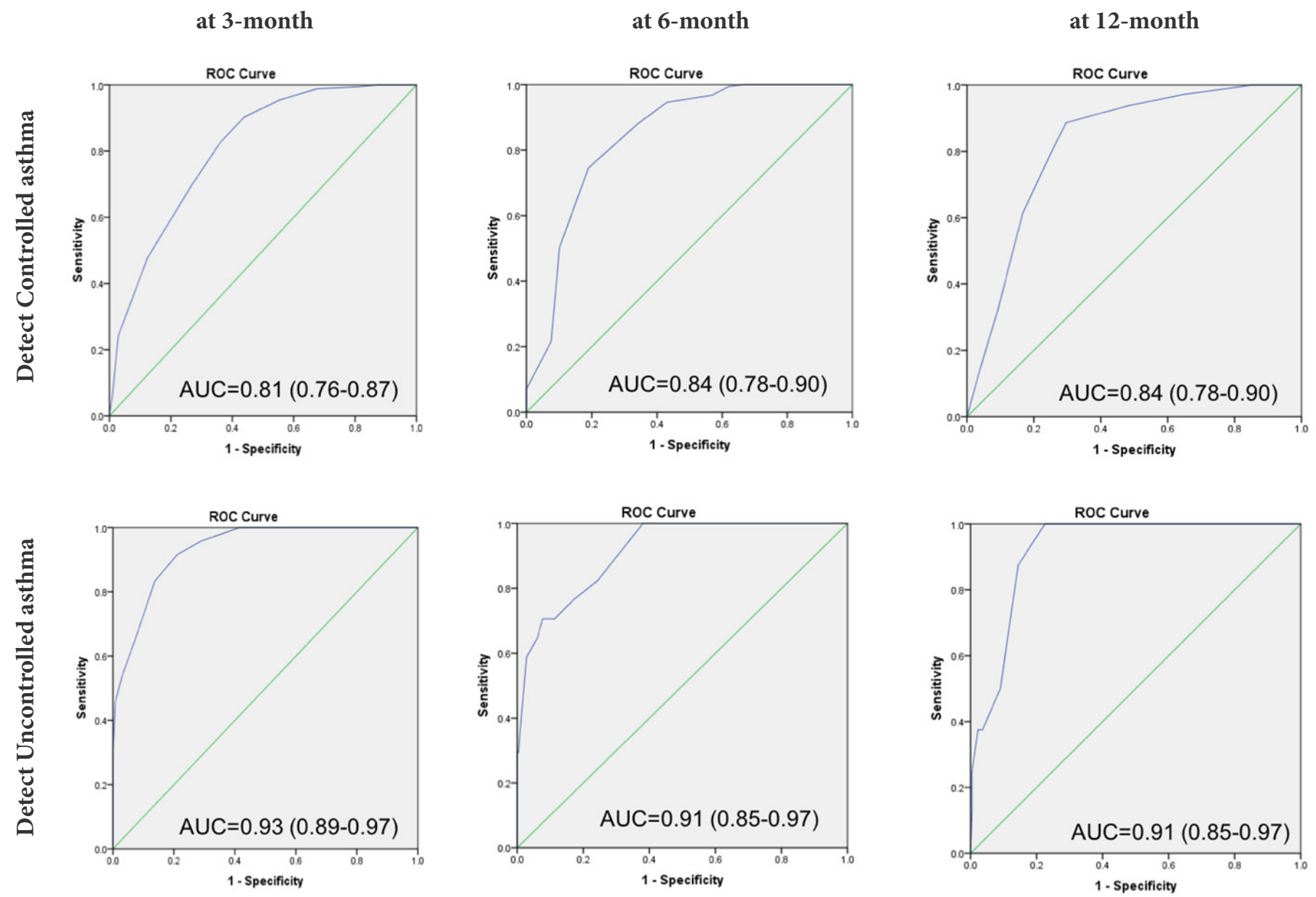

Figure 1. The receiver operating characteristic curves (ROC) of C-ACT score to detect controlled and uncontrolled asthma at 3-month, 6-month, and 12-month after initial treatment

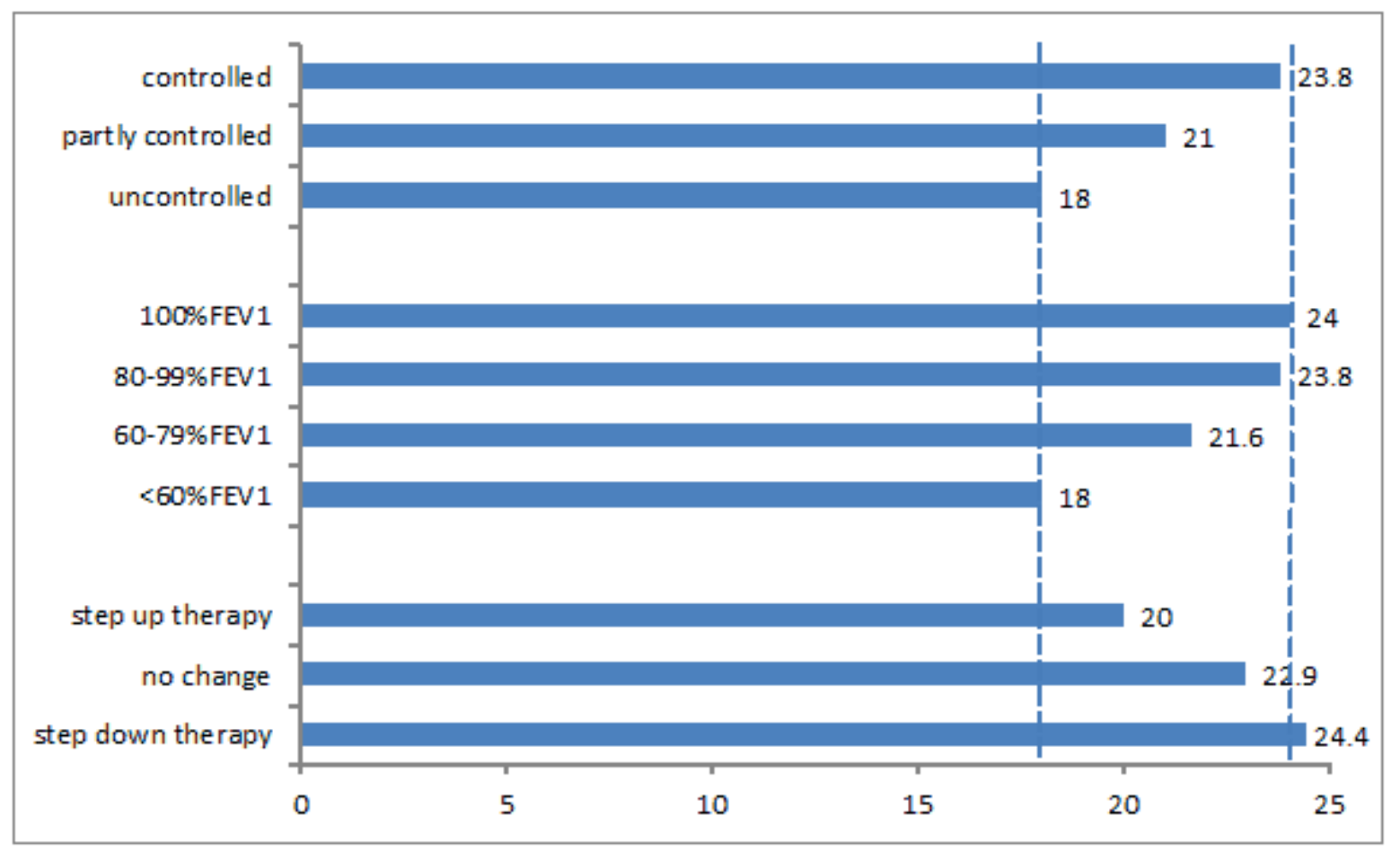

Figure 2. C-ACT cut-point score compared with clinical measures at 3-month visits. The cut-point scores for uncontrolled and controlled asthma levels were 18 and 23 , respectively. 
Table 4. C-ACT cut-point score compared with the level of asthma control according to the GINA guideline.

\begin{tabular}{|c|c|c|c|c|}
\hline & GINA guideline & C-ACT cut-point $23 \& 18$ & C-ACT cut-point $24 \& 18$ & C-ACT cut-point $24 \& 19$ \\
\hline & \multicolumn{4}{|c|}{ Step up therapy $(\mathrm{N}=73)$} \\
\hline Controlled & 4 & 18 & 13 & 13 \\
\hline Partly controlled & 46 & 35 & 40 & 30 \\
\hline \multirow[t]{2}{*}{ Uncontrolled } & 23 & 20 & 20 & 30 \\
\hline & \multicolumn{4}{|c|}{ No change (N=82) } \\
\hline Controlled & 48 & 56 & 46 & 46 \\
\hline Partly controlled & 33 & 13 & 23 & 30 \\
\hline \multirow[t]{2}{*}{ Uncontrolled } & 1 & 13 & 13 & 6 \\
\hline & \multicolumn{4}{|c|}{ Step down therapy $(\mathrm{N}=124)$} \\
\hline Controlled & 122 & 108 & 90 & 90 \\
\hline Partly controlled & 2 & 16 & 34 & 34 \\
\hline Uncontrolled & 0 & 0 & 0 & 0 \\
\hline
\end{tabular}

Abbreviations: C-ACT, Childhood Asthma Control Test; GINA, Global Initiative for Asthma.

$\leq 18$ demonstrated a sensitivity of $54.2 \%$, specificity of $96.9 \%$, PPV of $61.9 \%$, and NPV of $95.7 \%$.

Clinical correlation with C-ACT score was estimated by the level of asthma control according to the GINA guideline, the change in medical treatment, and percentage of predicted FEV1 value, as given in Figure 2. A C-ACT score of 23.8 represented controlled asthma whereas a score of 18 demonstrated uncontrolled asthma. Among patients with FEV1 less than $60 \%$ of the predicted value, the average mean C-ACT score was 18. For those with FEV1 more than $80 \%$ of the predicted value, which demonstrated controlled asthma, the average C-ACT score was 23.8 (Figure 2).

The aims of asthma treatment are to achieve asthma symptom control, maintain good quality of life, and minimize the medication needed for maintaining well-controlled asthma. After good asthma control had been achieved and maintained for about 3 months, a step down to a lower medication dose can be made as long as control of both symptoms and acute exacerbation is maintained. ${ }^{8-10}$ Figure 2 illustrates that step-up therapy is generally prescribed when C-ACT $=19.97$, no change when C-ACT $=22.92$, and step-down therapy when C-ACT = 24.39. In the step-up therapy group, an uncontrolled asthma group including 23 children was obtained, based on the level of asthma control according to the GINA guideline by specialist assessment; a group including 20 children was obtained by using a C-ACT score of 18 (Table 4). However, if a C-ACT score of 19 was considered, an uncontrolled group including 30 children requiring step-up therapy was obtained, which represents an overtreatment compared with a C-ACT score of 18.

For the controlled asthma group, using the ROC curve a C-ACT score cut-point of $\geq 23$ was chosen, consistent with step-down therapy. Specifically, no change and step-down therapy at the 3-month visit can be referred to with C-ACT scores of 22.9 and 24.4, respectively, as depicted in Figure 2.
Based on the level of asthma control by the GINA guideline, a group of 122 children with controlled asthma was obtained by specialist assessment whereas a group of 108 children was obtained using a C-ACT score of 23 in a step -down therapy group (Table 4). In contrast, a controlled group of 90 children in step-down therapy was found using a C-ACT score of 24 . However, a C-ACT score of more than 24 represented an overtreatment.

Moreover, we found that the C-ACT score cut-point for controlled and uncontrolled asthma groups at the 6-month visit had results similar to those at the 3 -month visit (Figure 1). Specifically, at C-ACT score $\geq 23$, we found $74.6 \%$ sensitivity and $81 \%$ specificity, representing controlled asthma. At C-ACT score $\leq 18$, we found $64.7 \%$ sensitivity and $93.9 \%$ specificity, demonstrating uncontrolled asthma. This result coincided with the correlation between C-ACT score and FEV1 at the 6-month visit, as given in Table 2. However, C-ACT score cut-points at the 1-year visit were not determined owing to a small number of patients with uncontrolled asthma at a follow-up visit 1year after treatment.

\section{Discussion}

The C-ACT has been translated into regional languages and used for assessing the level of control of asthma. Several studies have showed good clinical correlation with translated versions of the C-ACT. ${ }^{7-16}$ The GINA guideline is the gold standard for validating a translated version of the C-ACT. Pulmonary function tests have been used as a predictor of asthma control. FEV1 is one of the main methods of assessing pulmonary function as well as the level of asthma control, according to the GINA guideline.1,6 The C-ACT was developed to provide a simple self-administered questionnaire that is easy to use in primary care settings. A validated, translated version of the C-ACT is a useful tool for assessing asthma control. 
Statistical significance correlations between C-ACT score and FEV1 indicated that the C-ACT is a good predictor of asthma control. This study showed significantly positive correlations between C-ACT score and FEV1 at all visits. The results of our study were similar to those of other studies. ${ }^{7-8}$ Alvarez-Gutiérrez et al. found a correlation between the baseline FEV1 and C-ACT score $(\mathrm{r}=0.19$ and $\mathrm{p}<0.01){ }^{7}$ Chalise et al. found a significantly positive correlation between C-ACT score and FEV1 at enrollment $(r=0.772$ and $\mathrm{p}<0.001)$, the 3-month visit $(\mathrm{r}=0.815$ and $\mathrm{p}<0.001)$, and 6 -month visit $(\mathrm{r}=0.908$ and $\mathrm{p}<0.001){ }^{8}$ Finally, Lee et al. found that patients with a C-ACT score $>19$ had better pulmonary function test results, but there was weak correlation between pulmonary function test results and C-ACT score, with a correlation coefficient for FEV1 of 0.061 (95\% confidence interval: -0.022 to 0.049$){ }^{9}$

A study of the Tunisian Arabic version of the C-ACT administered to 51 patients found that a C-ACT score of 19 identified uncontrolled asthma (sensitivity $73.7 \%$ and NPV $86.5 \%$ ), and good correlation was found between C-ACT and clinical evaluation. ${ }^{10} \mathrm{~A}$ study of the Nepali version of the C-ACT among 65 patients found that a C-ACT score $\geq 19$ indicated controlled asthma (sensitivity 98.5\%, specificity 89.1\%, PPV 94.9\%, and NPV 96.6\%). ${ }^{8}$

The Spanish version of the C-ACT has been demonstrated to be are liable and valid questionnaire for evaluating asthma control, with a cut-point score $\geq 21$ indicating good asthma control (sensitivity ranging from $73 \%$ to $57.3 \%$, specificity ranging from $47.1 \%$ to $60.9 \%$, and correlation coefficient $\geq 0.85$ ). ${ }^{11}$ The previous study of Thai version of the C-ACT for 83 patients showed that the C-ACT score $<22$ determined uncontrolled asthma (sensitivity 73.9\%, specificity 96.7\%, PPV 93.2\%, NPV 93.2\% and AUC 0.91). However, the controlled or partly controlled asthma group was not mentioned. ${ }^{12}$ The Brazilian Portuguese version of the C-ACT showed statistically significance among GINA categories $(r \geq 0.3$ and $\mathrm{p}<0.01)$. Specifically, this indicated C-ACT scores for controlled $(22 \pm 2.9)$, partly controlled (20 \pm 4$)$, and uncontrolled (16.3 \pm 5.3$)$ asthma groups, which was similar to our study where the C-ACT scores were $\geq 23,19-22$, and $\leq 18$ for these groups, respectively. Furthermore, there was no significant correlation between C-ACT score and spirometry or exhaled nitric oxide $\left(\mathrm{r}=0.02\right.$ and $\mathrm{p}=0.866, \mathrm{r}=0.035$ and $\mathrm{p}=0.753$, respectively).$^{13}$

Most studies on a translated version of the C-ACT have found clinical correlations showing that the test can be used as a tool for assessing asthma control..$^{7-16} \mathrm{~A}$ Turkish version of the C-ACT was found to be an accurate and reliable tool for evaluating asthma control, with significant correlation between C-ACT and a physician's assessment of asthma control ( $r=0.65$ and $\mathrm{p}<0.001) .{ }^{14}$ The reliability and validity of the C-ACT in a population of Chinese children with asthma was demonstrated, with internal consistency reliability 0.741 at baseline and 0.759 at a follow-up visit. Reliability between the C-ACT and a specialist's rating of asthma control at baseline and follow-up was $r=0.546(\mathrm{p}<0.001) .{ }^{15}$ The original C-ACT study found a correlation between specialist-assessed change in therapy (step-up or step-down therapy) and C-ACT score $(\mathrm{p}<0.0001) .{ }^{4}$ Relevant to our study, a change in medication (step-up or step-down therapy) was related to the C-ACT score cut-point obtained from the ROC curve (Table 4).

On longitudinal analysis, we found that C-ACT was useful for predicting controlled asthma at 3-month and 6-month visits but not at 1 -year visits. This result is similar to a study by Leung et al., which found that the C-ACT can be useful to predict asthma exacerbation. Changes in C-ACT score are correlated with changes in asthma control status, DSS, and FEV1 ( $\mathrm{p}=0.019$, 0.034, and 0.020, respectively). ${ }^{16}$ Conversely, the Nepali C-ACT was found not to be useful for 6-month visits. ${ }^{8}$ At 1 -year visits in our study, we found a discrepancy between C-ACT correlated with FEV1 (Table 2) and C-ACT chosen by the ROC curve. This might be because at the 1-year visit, a group of uncontrolled asthma at 1-year visit decreased from 24 to 8 persons. Because this study is a follow-up study, we found some patients had lost to follow-up or some patients had better clinical outcome than the previous visit, which caused them to be discarded from an uncontrolled asthma group. Therefore, these reasons results in decreasing a sample size of the uncontrolled asthma group to 8 persons, which in turn can affect the accuracy of C-ACT chosen by the ROC curve and also leads to the discrepancy between the C-ACT correlated with FEV1 (Table 2) and the C-ACT chosen by the ROC curve at a 1-year visit.

In particular, the $\mathrm{C}$-ACT score cut-point for predicting uncontrolled asthma from the original C-ACT study was $\leq$ $19,{ }^{4}$ but that cut-point cannot predict partly controlled or uncontrolled asthma, according to the GINA guideline. For the C-ACT, scored >19 were also associated with "well controlled" or "totally controlled" asthma, scored of $<16$ were considered "poorly controlled" or "not controlled at all," and scored of 16-19 corresponded to "somewhat controlled" asthma. The discrepancy between our study and previous study may be because the patients and/or parent behavior in answering questionnaire is differences for each country, for example, in Thailand, people usually answer questionnaire positivity. Our study demonstrated the C-ACT score cutpoint for predicting the levels of asthma control in the GINA guideline and showed the correlation with FEV1. Therefore, the patient who had C-ACT score less than 23 had acute asthmatic attack once in one year and if the patient who had C-ACT score less than 18 had limited activity and exacerbation. The Thai version of the C-ACT can predict the level of asthma control without pulmonary function testing, which makes it easy to use in primary care settings.

\section{Conclusion}

The Thai version of the C-ACT is an accurate, simple, and useful tool for assessing asthma control among Thai children. A short, self-administered questionnaire for patients and caregivers is suitable for clinical practice settings. The correlation between $\mathrm{C}$-ACT and FEV1 shows that it coincides with the GINA guideline. Although the C-ACT score cannot compare with GINA guideline which is the gold standard for asthma diagnosis, it can help clinicians in a primary care setting to decide an appropriate medical treatment for asthma patients.

\section{Acknowledgments}

The authors are grateful to the children and parents who participated in this study. We thank Mrs. Thanavadee 
Meesathien of the Department of Pediatrics, Taksin Hospital, for administering the C-ACT questionnaires to our patients. We also thank the Faculty of Tropical Medicine, Mahidol University for support in proofreading the manuscript.

The authors declare that they have no conflicts of interest regarding the publication of this paper.

\section{References}

1. Global Initiative for Asthma. Global Strategy for Asthma Management and Prevention [Internet]. [place unknown]: Global Intiative for Asthma; c2016 [cited 2017 Apr 17]. Available from: http://www.ginasthma.org.

2. Pearce N, Ait-Khaled N, Beasley R, Mallol J, Keil U, Mitchell E, et al. Worldwide trends in the prevalence of asthma symptom: phase III of the International study of Asthma and Allergies in Childhood (ISAAC). Thorax. 2007;62:758-66.

3. Woolcock AJ, Yan K, Salmon C. Methods for assessing bronchial reactivity. Eur J Respir Dis. 1983;128:181-95.

4. Liu AH, ZeigerR, Sorkness C, Mahr T, Ostrom N, Burgess S, et al. Development and cross-sectional validation of the Childhood Asthma Control Test. J Allergy Clin Immunol. 2007;119:817-25.

5. Brooks SM, Bernstein IL, Raghuprasad PK, Maccia CA, Mieczkowski L. Assessment of airway hyperresponsiveness in chronic stable asthma. J Allergy Clin Immunol. 1990;85:17-26.

6. American Thoracic Society (ATS). Statement on standardization of spirometry - 1994 update. Am J Respir Crit Care Med. 1995;152:1107-39.

7. Alvarez-Gutiérrez FJ, Medina-Gallardo B, Pérez-Navarro P, Martin -Villasclaras JJ, Martin Etchegoren B, Romero-Romero B, et al. Comparison of the Asthma Control Test (ACT) with lung function, level of exhaled nitric oxide and control according to the GlobalInitiative for Asthma (GINA). Arch Bronconeumol. 2010;46:370-7.
8. Chalise SP, Bhatta NK, Singh RR, Prasad SM, Poudel P. Assessment of Control of Bronchial Asthma in Children Using Childhood Asthma Control Test. Indian J Chest Dis Allied Sci. 2014;56:75-8.

9. Lee MS, Kao JK, Lee CH, Tsao LY, Chiu HY, Tseng YC, et al. Correlation between pulmonary function and childhood asthma control test results in 5-11 year old children with asthma. Pediatr Neonatol. 2014;55:218-24.

10. Ouali H, Zneidi M, Berraiss A, Ben Safta B, Ammar J, Sellami A, et al Childhood asthma control test: Validation of Arbic Tunisian dialect version in 51 patients. Eur Respir J. 2012;40 Suppl 56:S3970.

11. Pérez-Yarza EG, Castro-Rodriguez JA, Villa Asensi JR, Garde Garde J, Hidalgo Bermejo FJ. Validation of a Spanish version of the childhood asthma control test (SC-ACT) for use in Spain.Anales de Pediatria. 2015;83:94-103.

12. Dardaranonda B. An Evaluation of Thai-Translated Version of The Childhood Asthma Control Questionnaire (C-ACT) and The Composite Asthma Severity Index (CASI) Among Thai Asthmatic Children [abstract]. J Allergy Clin Immunol. 2014;133(2 Suppl):SAB6.

13. Oliveira SG, Sarria EE, Roncada C, Stein RT, Pitrez PM, Mattiello R Validation of the Brazilian version of the childhood asthma control test (c-ACT). Pediatr Pulmonol. 2016;51:358-63.

14. Sekerel B, Soyer O, Keskin O, Uzuner N, Yazicioglu M, Kiliç M, et al. The reliability and validity of Turkish version of Childhood Asthma Control Test. Qual Life Res. 2012;21:685.

15. Chen HH, Wang JY, Jan RL, Liu YH, Liu LF. Reliability and validity of childhood asthma control test in a population of Chinese asthmatic children. Qual Life Res. 2008;17:585-93.

16. Leung TF, Ko FW, Bhome A, Wong GW, Li CY, Hui DS, et al. Predicting change in clinical status of young asthmatics: clinical scores or objective parameters? Pediatric Pulmonol. 2009;44:442-9. 